Issued by Sandia National Laboratories, operated for the United States Department of Energy by Sandia Corporation.

NOTICE: This report was prepared as an account of work sponsored by an agency of the United States Government. Neither the United States Government, nor any agency thereof, nor any of their employees, nor any of their contractors, subcontractors, or their employees, make any warranty, express or implied, or assume any legal liability or responsibility for the accuracy, completeness, or usefulness of any information, apparatus, product, or process disclosed, or represent that its use would not infringe privately owned rights. Reference herein to any specific commercial product, process, or service by trade name, trademark, manufacturer, or otherwise, does not necessarily constitute or imply its endorsement, recommendation, or favoring by the United States Government, any agency thereof, or any of their contractors or subcontractors. The views and opinions expressed herein do not necessarily state or reflect those of the United States Government, any agency thereof, or any of their contractors.

Printed in the United States of America. This report has been reproduced directly from the best available copy.

Available to DOE and DOE contractors from

Office of Scientific and Technical Information

P.O. Box 62

Oak Ridge, TN 37831

Prices available from (703) 605-6000

Web site: http://www.ntis.gov/ordering.htm

Available to the public from

National Technical Information Service

U.S. Department of Commerce

5285 Port Royal Rd

Springfield, VA 22161

NTIS price codes

Printed copy: A03

Microfiche copy: A01

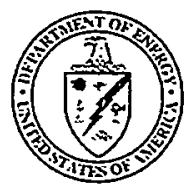




\section{DISCLAIMER}

Portions of this document may be illegible in electronic image products. Images are produced from the best available original document. 
SAND 99-2848

Unlimited Release

Printed November 1999

\title{
Computer Network Availability at Sandia National Laboratories, Albuquerque NM: Measurement and Perception
}

\author{
Lawrence Tolendino \\ Advanced Network Integration Department \\ Spencer Nelson \\ Telecommunications Operations Department \\ Sandia National Laboratories \\ P.O. Box 5800 \\ Albuquerque, NM 87185-0806
}

\begin{abstract}
The desire to provide a measure of computer network availability at Sandia National Laboratories has existed for a long time. Several attempts were made to build this measure by accurately recording network failures, identifying the type of network element involved, the root cause of the problem, and the time to repair the fault. Recognizing the limitations of available methods, it became obvious that another approach of determining network availability had to be defined. The chosen concept involved the periodic sampling of network services and applications from various network locations. A measure of "network" availability was then calculated based on the ratio of polling success to failure. The effort required to gather the information and produce a useful metric is not prohibitive and the information gained has verified long held feelings regarding network performance with real data.
\end{abstract}




\section{Introduction}

The desire to provide a measure of computer network availability at Sandia National Laboratories has existed for a long time. The desire was born of the perception that we should be able to objectively quantify the "goodness" of our computer network's performance as it relates to our customers. In addition, the network operation staff desired to use a measure of availability to record progress in improving data communications network performance. As a by-product of the measurement, the staff could also identify troublesome network elements.

\section{History}

Several attempts were made to build this measure by accurately recording network failures, identifying the type of network element involved, the root cause of the problem, and the time to repair the fault. The process of gathering the required information involved many of the staff and therefore contained much variability in detail, quality, and completeness. Once the information was gathered, the analysis proved to be very labor intensive and the results were often ambiguous. The results just did not seem to justify the efforts. In addition, such analysis did not record the network impact of the failure and was not capable of being converted into a measure of network availability.

One was forced to conclude that in a local area network of over 500 switches, hubs, and routers it was not practical to determine a measure of network availability by recording the performance of all the individual network elements. In addition, the customer's perception of network availability is formed by the interplay of the network elements, network services, and applications. In particular, the perception is based on the availability of not only the customer's network connection but also network services such as DNS (Domain Name Services) or applications such as E-mail.

Recognizing the limitations outlined above, it became obvious that another method of determining network availability had to be defined.

\section{A New Definition of Network Availability}

The definition of network availability desired would meet the needs of the network operations staff, provide a measure network availability that could be related to the customer's experience using the network, and be relatively easy for everyone to interpret. In the classic sense availability (A) is defined as: $A=(M T B F-M T T R) / M T B F$ where MTBF is defined as the mean time between failures and MTTR is the mean time to repair. Such a definition is very useable when one is concerned with a single element or a system of elements with very well defined interrelationships. In a complex system composed of network elements, network services, and applications the classic definition of availability is difficult to apply. Once the limitations of applying classical techniques 
were appreciated other approaches to determining a measure of network availability were explored.

The first concept to be explored was based on the strategy of categorizing each network element by the impact the element's failure would have on the customers. While such categorization appeared feasible, determining the customer impact proved elusive. Even determining how many people might be affected by the failure of a single element is a difficult and imprecise task. In addition, the Sandia National Laboratories computer networks are so dynamic that the categorizations and impact ratings would probably change during the measurement periods. At best this concept, if implemented, would have been able to provide a measure of availability from a network viewpoint but would not provide such a measure from the customer's viewpoint. Given this concept's limitations, it did not seem worthwhile to pursue it any further.

Recognizing these limitations Pete Hamilton suggested that we consider defining a statistical measure of network availability. Briefly, the concept involved the periodic sampling of network services and applications from various network locations. A measure of "network" availability would then be calculated based on the ratio of polling success to failure. After some research into the tools available it was determined that the WhatsUp network-monitoring tool (software from Ipswitch, Inc.) could be used to calculate such a measure of network availability for any network service or application from any arbitrary location in the network. To simplify the task of calculating the Sandia National Laboratories restricted network (SRN) availability DNS (Domain Name Service) servers were chosen as the polling targets.

The DNS servers were chosen as the polling target because of their highly reliable and well-known performance characteristics. Therefore, DNS system performance would not affect the measurements. It was decided to accomplish the availability measurement by periodically polling the network SRN DNS servers using both an ICMP (ping) and DNS poll. In this way one can determine that not only is the target system running but the DNS service is also running. By implementing several WhatsUp polling stations throughout the Sandia National Laboratories network one can determine a measure of network availability.

\section{Implementation}

Four WhatsUp network-monitoring systems located in buildings $6585,870,880$, and 891 were connected to the SRN and began gathering data in March 1999. The monitors were configured to poll a selection of network elements, network services and applications with appropriate probes to gain a broad view of network performance from the customer's perspective. For the purposes of determining network availability, two of the DNS servers, NS3 and NS4, were polled with an ICMP and DNS probe at one-minute intervals. The polling was limited to normal Sandia National Laboratories business hours, Monday - Friday, 0730 to 1700 hours, in order to eliminate the impact of normal maintenance operations from the measurement. The statistical reports generated by the WhatsUp systems were queried via WEB access at the end of the month to record the polling success rate. Once the results were recorded the counts were reset to zero. 
After a baseline of SRN availability is created from several months of these measurements, Sandia plans to move some of the probes to other locations in the SRN. Moving the monitoring systems will allow network operations to determine if there are any variances in performance throughout the SRN.

\section{Initial Results}

The initial results for WhatsUp measurements are presented below. The first graph shows the measure of network availability for the month of May 1999 while the second graph shows the trends of the measurements over a six-month interval.

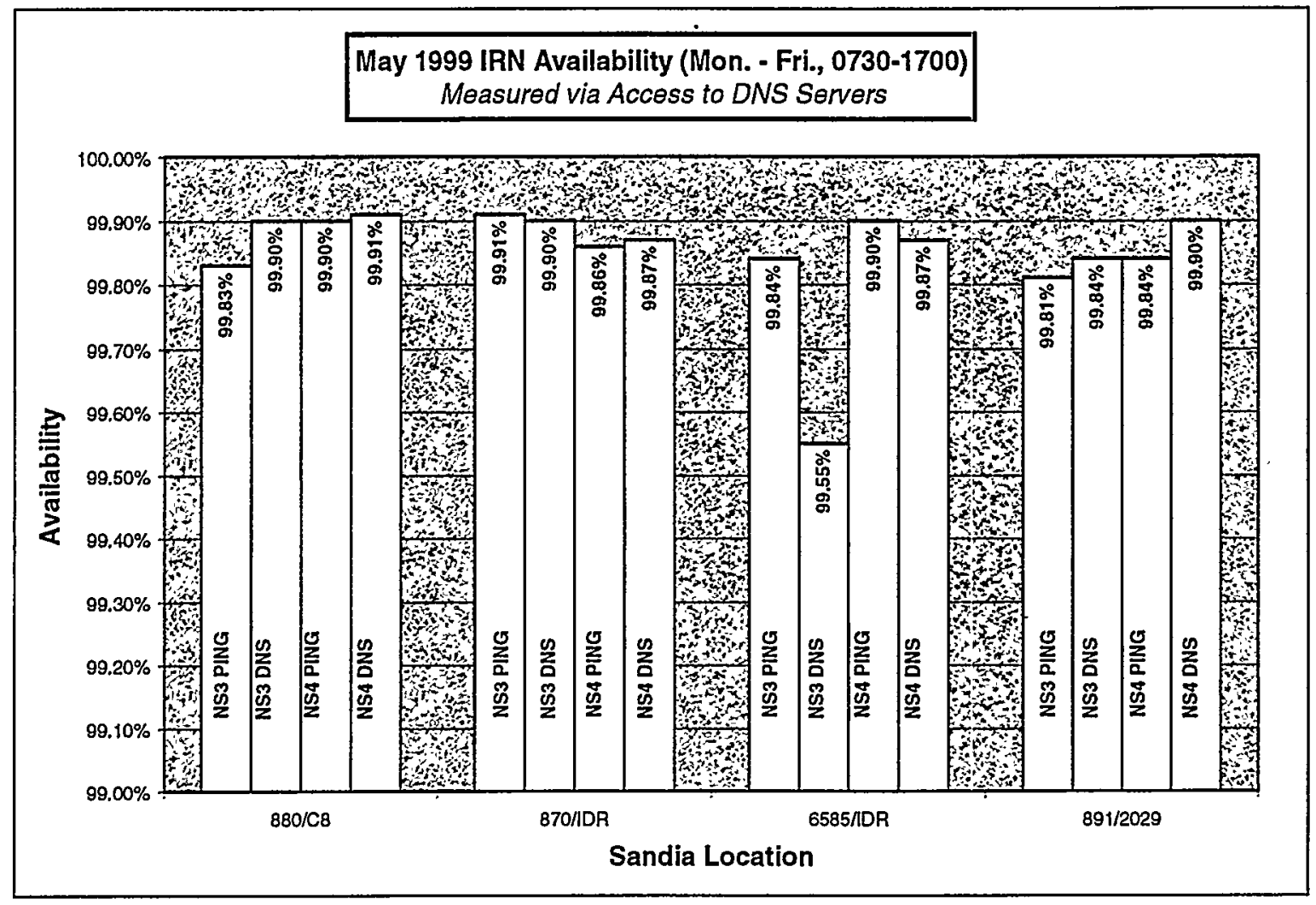

In the above graph the availability of each DNS server is recorded via the ICMP poll and the DNS poll for each WhatsUp system. However, the above graph does not reflect any changes. In order to determine the monthly trends, average ICMP and DNS responses were calculated across the four WhatsUp locations and plotted in the following graph. 


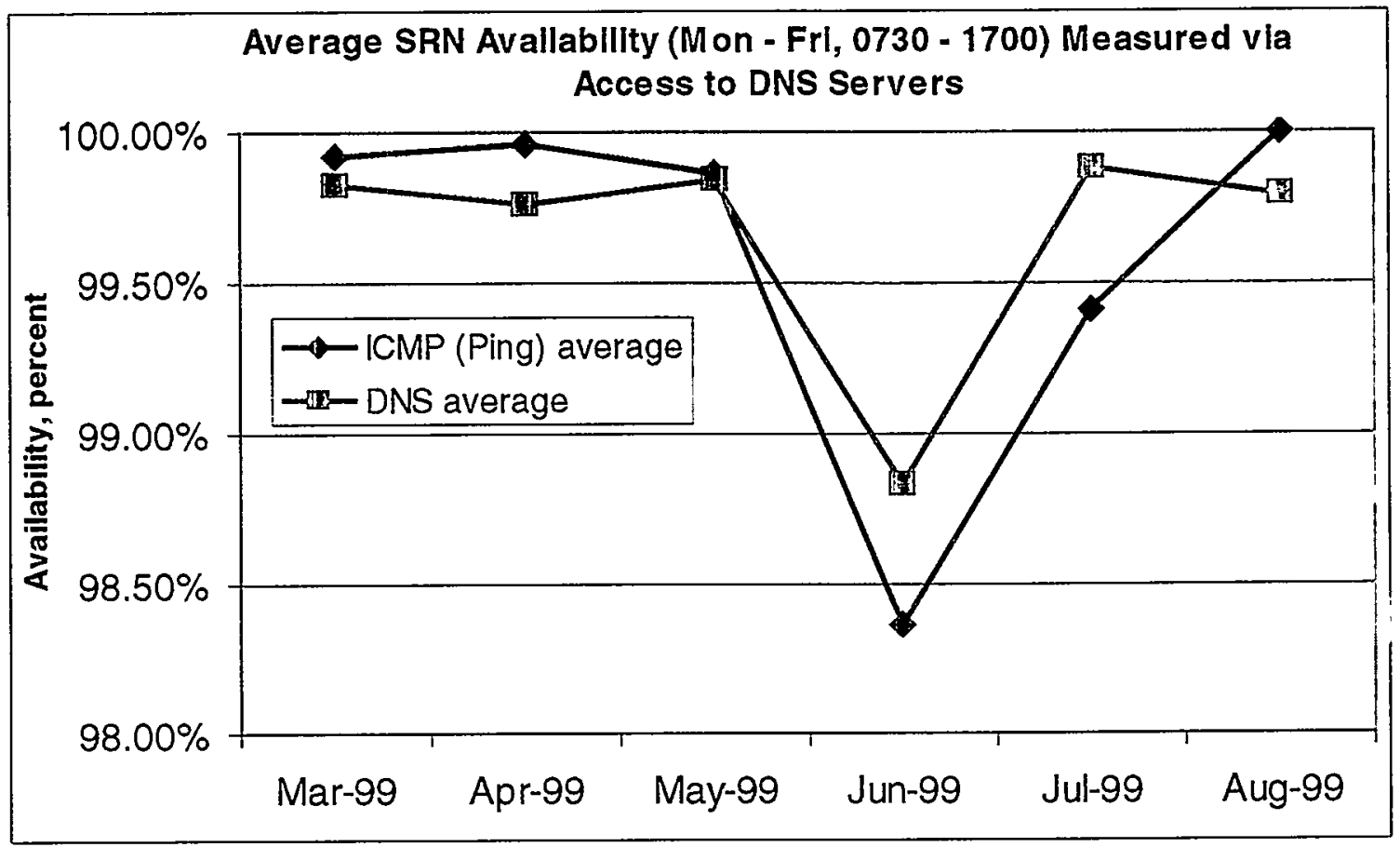

\section{Limitations}

The astute reader will note that the availability measures reported in this paper will not necessarily reflect network availability as perceived by the Sandia National Laboratories customers. The purpose of generating a measure of network availability was to reflect the availability as perceived by the customer AND provide a metric that would allow network operations staff to measure network performance improvements. The measures defined here will satisfy the second goal but not the first. Remember that customer perceived SRN availability is the result of the interactions of network elements, network services, and applications. Indeed, while the network availability measures detailed above $(99 \%+)$ look very comforting to the network operations staff, the customer perceived availability will be much less. How do we know this? The plethora of measurements made by the WhatsUp systems give us the information.

A brief glance at the following graph shows the differences in availability measured for data network elements and other network services. 


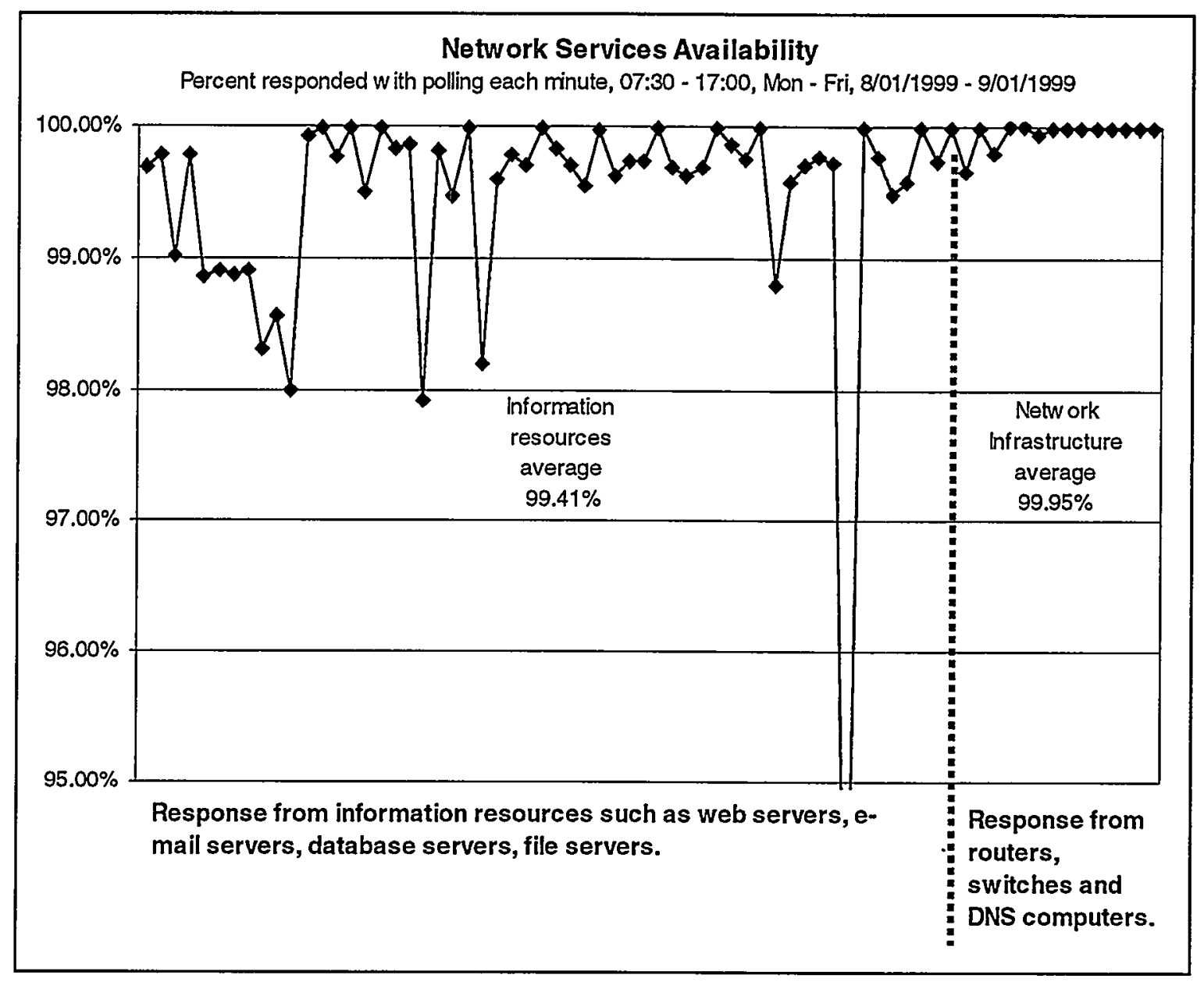

The chart above titled "Network Services Availability" compares the response from network infrastructure elements (routers, switches, and DNS computers) with the response from information resources (a list of web servers, e-mail servers, database servers, and file servers). Polls are made once per minute and include ICMP (ping) as well as interrogations of one of more TCP ports appropriate to the services operating on the server. For example, for a simple web server, the http port (port 80 ) is tested for response. If a response is not received within a specified time interval (normally 2 seconds) then that test is considered failed for that poll interval. This type of testing gets us closer to the perceived availability of a typical person using a multi computer application, but of course it still does not include the actual effects of programs running on either the servers or client computer.

There can be a major difference between the availability of applications and the availability of the data transmission "network". Therefore, the customer can perceive an availability that is significantly lower than the network availability defined in previous sections of this paper. Using measurements provided by monitoring stations can provide a method of tracking improvements in application performance. Indeed, for some applications we have used programmed client computers as robots to measure overall application availability. 
For a more complete discussion of the complexity of measuring application performance please see the report SAND99-2001, Unlimited Release, Printed August 1999, "Applications Analysis: Principles and Examples from Various Distributed Computer Applications at Sandia National Laboratories, New Mexico", by Dennis Bateman, David Evans, Dal Jensen and Spencer Nelson, Department Sandia National Laboratories, P.O. Box 5800, Albuquerque, NM 87185-0807.

URL: http://infoserve.sandia.gov/sand_doc/1999/992001.pdf

\section{Conclusions}

While we were not able to implement a measure of network availability that met both of our goals, the statistical measure of network availability defined has proved to be both useful and practical. The effort required to gather the information and produce a useful metric is not prohibitive and the information gained has verified long held feelings regarding network performance with real data.

First, the availability of the data transmission network and network services is really very good, usually exceeding $99 \%$

Second, it is apparent from the monthly measurements that there are some differences based on location within the network. Measurements from building 6585, which is in Tech Area V, have consistently showed lower availability than those sites in Tech Area I. That result has been consistent for each month measured.

Third, we must work to improve the time required to repair a network outage. One can see the impact that one significant network outage has on general network availability in the graph of monthly averages. When a critical network element failed in June, the twohour repair time caused network availability to fall precipitously. It is apparent that to achieve greater than $99 \%$ network availability consistently Sandia National Laboratories must be able to improve the time to repair.

Forth, it would not be effective to focus on improving the network and network services availability without making improvements to other facets of applications. Expending significant resources improving the data transmission network will not necessarily change the customer's perception. However, Sandia does appear to be improving overall application availability. Being able to measure availability, along with the components of availability, allows resources to be directed to improving the areas that need it the most.

Last, while we are contemplating our next steps, it would be prudent to move some of the probes to other locations in the SRN to gain a more comprehensive view of SRN network availability. 


\begin{tabular}{lll}
\multicolumn{3}{l}{ DISTRIBUTION } \\
1 & 0801 & Melissa Murphy, 4600 \\
1 & 0806 & Michael Vahle, 4600 \\
5 & 0806 & Lawrence Tolendino, 4616 \\
1 & 0806 & Leonard Stans, 4616 \\
1 & 0803 & L. Herbert Pitts, 4900 \\
1 & 0812 & Dennis Bateman, 4614 \\
1 & 0812 & David Evans, 4614 \\
1 & 0812 & Dal Jensen, 4614 \\
5 & 0812 & Spencer Nelson, 4614 \\
1 & 0812 & Michael Sjulin, 4614 \\
1 & 0812 & Judy Chavez, 4614 \\
1 & 0812 & Joseph Maestas, 4614 \\
1 & 0812 & Pat Manke, 4614 \\
1 & 0812 & Bruce Whittet, 4614 \\
1 & 1204 & Peter Hamilton, 5932 \\
1 & 9018 & Central Technical Files, 8940-2 \\
2 & 0899 & Technical Library, 4916 \\
1 & 0612 & Review \& Approval Desk, 4912 \\
& & For DOE/OSTI
\end{tabular}

